Morrison, 1992) and am now in the zolpidem/ zopiclone zone (e.g. Lader, 1997; Gericke \& Ludolph, 1994 ) and would have valued rather more discussion on non-pharmalogical interventions, possibly along the line of Morin et al (1994). May I ask for an article on non-pharmacological treatment of sleep disorders in adults, particularly those with substance misuse problems?

Gericke, C. A. \& Ludolph, M. D. (1994) Chronic abuse of zolpidem. Jourmal of the American Medical Association, 272, 1721-1722.

Lader, M. (1997) Is there any dependence and abuse potential? Journal of Neurology, 244 (suppl. 1), S18-S22.

Morin, C. M., Culbert, J. P. \& Schwartz, S. M. (1994) Nonpharmalogical interventions for insomnia. American Journal of Psychiatry, 151, 1172-1180.

Ruben, S. M. \& Morrison, C. L. (1992) Temazepam misuse in a group of injecting drug users. British Journal of Addiction, 87, 1387-1392.

Wilson, S. \& Nutt, D. (1999) Treatment of sleep disorders in adults. Advances in Psychiatric Treatment, 5, 11-18.

Dr Andrew Robinson Consultant Psychiatrist, Substance Misuse Service, Fulton Clinic, Royal Cornhill Hospital, Aberdeen AB25 2ZH

\section{Dentistry and psychiatry}

Sir. I find it astonishing that in a recent issue of APT the paper on 'understanding the importance of oral health in psychiatric patients' completely ignored the problem of bruxism or tooth grinding - arguably one of the most common problems linking dentistry with psychiatry.

Cormac, I. \& Jenkins, P. (1999) Understanding the importance of oral health in psychiatric patients. Advances in Psychiatric Treatment, 5, 53-60.

Dr Raj Persaud Consultant Psychiatrist, Westways Rehabilitation Unit, 49 St James's Road, West Croydon, Surrey CR9 2RR

Changes in suicidal ideation and psychomotor retardation during electroconvulsive therapy

Sir: Porter \& Ferrier (1999) describe the clinical impression that, during the early stage in treatment of depression, a reduction in psychomotor retardation may lead to patients with depression acting on suicidal thoughts. Consequently, this early stage in treatment is often viewed as the time of highest suicide risk. Slater \& Roth (1969) and Himmelhoch (1987) raised similar concerns. However, as Mann \& Kapur (1991) observed, no data exist to confirm or refute this.

If this stage of treatment of depression is the time of highest risk, one possible explanation is that the reduction in suicidal ideation lags behind the improvement in psychomotor retardation. We were interested in this issue with particular respect to electroconvulsive therapy (ECT) and prospectively studied changes in suicidal ideation and psychomotor speed in a group of 20 in-patients meeting DSM-IV criteria for major depression who were being treated with ECT. Prior to starting ECT and on the day after each application of ECT, subjects were assessed using the Montgomery-Asberg Depression Rating Scale (MADRS; Montgomery \& Asberg, 1979), a modified version of the Suicidal Ideation Scale (SIS; Beck et al, 1979) and a test of psychomotor speed derived from a cognitive testing battery by Coughlan \& Hollows (1985).

We studied changes in SIS and psychomotor speed scores over the first six applications of ECT in the subgroup of 16 patients who recovered or improved. With the overall changes expressed as percentages, a $92 \%$ reduction in suicidal ideation was observed during the first three treatment sessions, with only an $8 \%$ reduction over the next three sessions. Psychomotor speed improved $64 \%$ over the first three sessions and by $36 \%$ over the next three sessions.

Our results are similar to those reported by Rich et al (1986), who measured changes in the suicide ideation, decreased energy and decreased work/ activities subscores of the Hamilton Rating Scale for Depression in 37 patients receiving ECT. They reported that the mean maximal improvements in suicidal ideation occurred significantly sooner, and after significantly fewer treatments, than the improvements in energy symptoms.

It would be naiive to suggest that suicide risk could simply be equated with the balance between suicidal ideation and psychomotor retardation, as various other factors unique to the individual patient are likely to affect any decision to attempt suicide. However, the hypothesis that improvements in suicidal ideation might lag behind improvements in psychomotor retardation appears to be incorrect - the opposite is the case. If the early stages of ECT treatment are really the time of highest risk, this may simply be due to suicidal ideation being at its highest then. Risk of suicide should be considered throughout the course of ECT while there is any evidence to suspect continuing suicidal ideation. What our study and that of Rich et al (1986) demonstrate, however, is that ECT is a remarkably effective treatment for rapidly reducing suicidal ideation in depression.

Beck, A. T., Kovacs, M. \& Weissman, A. (1979) Assessment of suicidal intention: The Scale for Suicide Ideation. Journal of Consulting and Clinical Psychology, 47, 343-352.

Coughlan, A. K. \& Hollows, S. E. (1985) The Adult Information Processing Battery. Leeds: St James's University Hospital. Himmelhoch, J. M. (1987) Lest treatment abet suicide. Journal of Clinical Psychiatry, 48, 44-54.

Mann, J. J. \& Kapur, S. (1991) The emergence of suicidal ideation and behaviour during antidepressant pharmacotherapy. Archives of General Psychiatry, 48, 1027-1033. 